\title{
Function and morphology in macular retinoschisis associated with optic disc pit in a child before and after its spontaneous resolution
}

\author{
Anna A. Polunina $\cdot$ Margarita G. Todorova $\cdot$ \\ Anja M. Palmowski-Wolfe
}

Received: 11 November 2011 / Accepted: 26 January 2012/Published online: 10 February 2012

(C) Springer-Verlag 2012

\begin{abstract}
Optic disc pit (ODP) is a rare congenital defect within the optic nerve head. Macula elevation associated with ODP develops in 75-93\% of the adult patients. Macular involvement in children with optic disc pit is rare, and only a few cases have been published to date. In the present case, we have observed morphology and function of the central retina in a child with ODP-associated macular detachment and following its spontaneous resolution. An 8-year-old white boy diagnosed with a macular detachment in an eye with an ODP. Optical coherent tomography (OCT) and multifocal electroretinography (mfERG), as well as visual acuity and visual field, were performed in the follow-up of the unilateral schisis-like retinal detachment. A large retinoschisis associated with ODP in a child showed a tendency to spontaneously resolve at 3 months, which was confirmed on OCT. At this time, an mfERG revealed markedly reduced responses. Despite morphologic reattachment at follow-up and improvement in visual
\end{abstract}

This report has been partly presented as a poster at the Conference "Developments in Visual Science: Function meets Morphology" on June 9-11, 2010, Munich, Germany.

A. A. Polunina $(\bowtie) \cdot$ M. G. Todorova

A. M. Palmowski-Wolfe

Department of Ophthalmology,

University of Basel,

Mittlere Strasse 91,

4031 Basel, Switzerland

e-mail: PoluninaA@uhbs.ch acuity, increased mfERG responses were still not the same as in the fellow healthy eye. In contrast to the OCT which is very helpful to assess the extent of the neurosensory detachment, the mfERG offers an additional tool for follow-up of retinal function in this disorder. The good visual outcome in our patient shows that in the presence of residual retinal function on mfERG and in the absence of further lesions on OCT, follow-up is a valid option in children with an ODP-associated macular detachment.

Keywords Optic disc pit - Macular retinoschisis . Child $\cdot$ mfERG $\cdot$ OCT

\section{Introduction}

Optic disc pit (ODP) is a rare congenital defect within the optic nerve head, which was first described by Wiethe in 1882. Macula elevation associated with ODP develops in $75 \%$ [1] to $93 \%$ [2] of the adult patients. Lincoff $\mathrm{H}$. et al. have reported that this macular "detachment" is frequently a retinoschisis without a true retinal detachment because of the schisis-like nature of the inner retina [3, 4]. Although some detachments resolve spontaneously, others persist for years [5]. Macular involvement in children with an optic disc pit has been reported only in a few cases [6-8].

Several treatment options have been suggested in the literature with variable morphologic outcome in 
children and adults: photocoagulation, macular buckling, pars plana vitrectomy, gas tamponade [6]. The prognosis for return of vision after serous macular detachment is also variable.

The pathogenesis of this maculopathy is still unknown. Recent studies with optical coherence tomography (OCT) have clarified its morphological appearance. To date, we found only two reports describing retinal function with electroretinography and both of these are following surgical treatment $[1,7]$. These reports could not be compared with the results of the few case reports where spontaneous resolution of macular detachment occurred, because they do not report electrophysiology.

We would like to present a first case of morphological and functional evaluation of spontaneous macula reattachment in a child with an optic disc pit including optical coherence tomography (OCT) and multifocal electroretinography (mfERG).

\section{Case report}

We report on an 8-year-old Caucasian boy with an optic pit on the left eye, who was referred for evaluation of anisometropic amblyopia. His cycloplegic refraction was right eye $+1.75-0.5$ A130, left eye +3.5. On examination, his best corrected Snellen visual acuity was right eye -1.0 , left eye -0.5 . Near acuity of his right eye was 0.8 and his left eye only achieved 0.25 .

The anterior segment examination of both eyes was unremarkable. Fundus examination of the right eye was unremarkable. The left eye revealed an optic nerve head with a grey round excavation on the temporal side, containing an optic disc pit. A light reflex close to the vascular arcades raised the suspicion of a detachment of the neurosensory retina (Fig. 1a). No evidence of a macular hole was determined. Visual field examination using the Octopus G2 dynamic program revealed a central scotoma in the left eye. Macular schisis was confirmed on OCT (Fig. 2a). Fluorescein angiography revealed no leakage from the optic disc pit. A mild hypofluorescence marks the adjacent zone of serous detachment (Fig. 3).

The patient was asked to wear an optical correction to correct for the anisometropia. Occlusion treatment for $2 \mathrm{~h}$ per day was recommended to treat a possible concurrent refractive amblyopia, as the onset of disease was unknown. On follow-up 3 months later, the "subretinal cavity" was markedly improved on ophthalmoscopy and OCT (Fig. 2b). Distance acuity of the left eye also improved to 0.8 , near acuity to 0.6 . Visual field examination showed improvement in the central sensitivity and slight loss of sensitivity in the inferior-nasal segment (MD = 3.1, Octopus 101).

Because of the young age of the patient, the improvement in visual acuity and the partial retinal reattachment, no surgical procedure was undertaken, but a conservative approach of follow-up was chosen.

At this point in time, electrophysiology was performed. Pattern visual evoked potentials showed normal amplitudes of both eyes but increased latencies in the left eye. Amplitudes and latencies of the scotopic and photopic electroretinography were within the normal range on both eyes. The electrooculography was normal in the right eye but slightly pathologic in the left eye (Arden Quotient right eye 2.2 and left eye 2.6 (norm 1.5-2.5)), suggesting some minor secondary impairment of the retinal pigment epithelium.

Figure 4 shows the results of a multifocal electroretinogram (mfERG) which was performed according to the ISCEV guidelines [9] (VERIS $^{\mathrm{TM}}$, FMS III stimulator, DTL electrode, $\mathrm{m}=2^{15}, \mathrm{Lmx} 200 \mathrm{~cd}$, $\mathrm{Lmin}<1 \mathrm{~cd}$ ). It was normal in the right eye. In the left eye, good response waveforms were present in the peripheral responses (Fig. 4a). However, amplitudes were markedly reduced and latencies increased, especially in the central $30^{\circ}$ (Fig. $4 \mathrm{~b}, \mathrm{c}$ ). At the follow-up examination 4 months later, the patient showed an improvement on OCT. Distance visual acuity remained stable at 0.8 . Serous detachment decreased to a superior nasal parafoveal location (Fig. 2c).

Distance visual acuity improved to 1.0 on the left eye and stayed unchanged till the last follow-up 2 years after the initial visit. His cycloplegic refraction on the left eye changed to $+3.0-0.75$ A0. The decrease in hyperopia indicates the increase in the axial distance of the eye due to the decrease in retinal thickness. The OCT picture demonstrated a very local schisis-like splitting superior nasal in the perifoveal area which had continued to decrease over time (Fig. 2d-f). Fundus biomicroscopy revealed the dithering of the perifoveal superior and nasal schisis reflex (Fig. 1b).

At this point in time (15 months after the initial electrophysiological examination), the mfERG was repeated. In agreement with the good visual and 


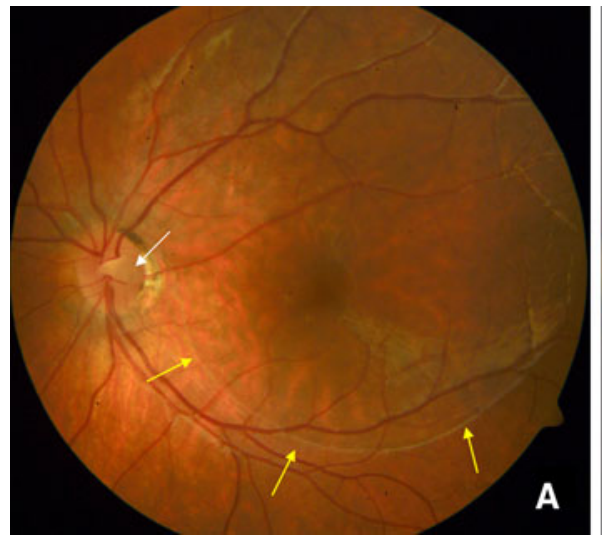

Fig. 1 a Retinal image of the left eye at the initial visit showing a temporal congenital pit of the optic disc (white arrow). A light reflex close to the vascular arcades demarks serous elevation of the retina (yellow arrows). b Retinal image at 21 months

morphologic recovery, there was a marked improvement in the central waveforms. However, amplitudes and latencies did not reach normal levels when compared to the right eye (Fig. 5a, b).

\section{Discussion}

Serous retinal detachments may be associated with optic disc pits. While they may occur as early as 6 years of age and as late as the ninth decade of life, they are most frequently observed in early adulthood [5]. In our case, we describe the rare condition of a unilateral optic disc pit with an associated schisisrelated macular detachment in an 8-year-old boy.

Macular lesions, such as macular serous detachment, cystic degeneration and degenerative pigment changes, are now known as common complications of an optic disc pit, which lead to poor visual acuity [6]. In addition, hyperopic refraction induced by an elevated macula could potentially cause additional amblyopia in children.

Recent OCT findings have revealed that the macular detachment associated with an optic disc pit consists of a bilaminar structure, and there is a connection between the pit and the intraretinal schisis [4].

It has been hypothesised that fluid from the pit causes an elevation of the nerve fibre layer leading to a schisis-like inner layer separation. When an outer layer macular hole develops beneath the inner layer,

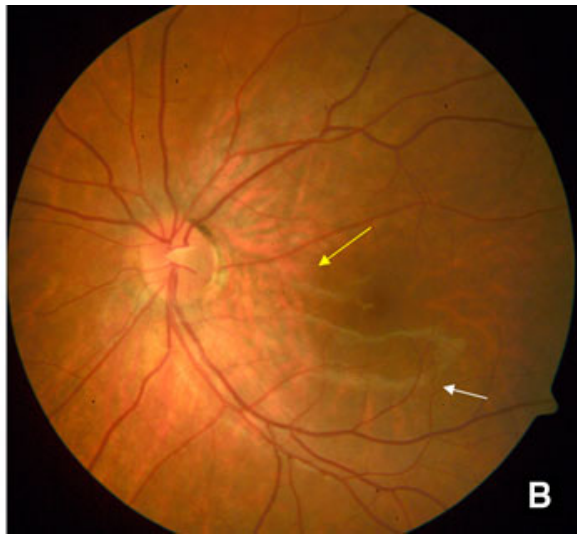

follow-up: An irregular light reflex is still present in the superior nasal perifoveal area (yellow arrow). Altered retinal reflexes are seen in the area of spontaneous reattachment (white arrow)

outer layer retinal detachment occurs around the macular hole and finally detachment increases $[3,10]$. Various possible origins of the fluid have been proposed in the literature: fluid from the vitreous cavity, cerebrospinal fluid, fluid from leaky blood vessels at the base of the pit and fluid from the orbital space surrounding the dura [11-15].

Several medical and surgical methods of treatment in the situation of macular evaluation have been described in the literature. The overall prognosis, however, remains poor particularly when there has been a long-standing sensory retinal detachment [6]. Conservative management used to be the initial recommendation, as $25 \%$ of ODP maculopathies resolve spontaneously [10]. In the past, retinal specialists performed laser photocoagulation if there was no resolution of the detachment within 3 months. Nowadays, if the fluid does not resolve within 3 months of treatment, a more aggressive surgical approach such as posterior vitreous detachment (PVD) and pars plana vitrectomy is taken [10].

In young patients especially in children, the connection of the posterior vitreous membrane to the retina is extremely strong, and a surgical approach such as pars plana vitrectomy is extremely difficult. A vitrectomy can lead to complications such as cataract or postvitrectomy retinopathy. However, a vitreoretinal approach in combination with laser photocoagulation and gas tamponade has been reported in children with a maculopathy associated with optic disc pit: In these 

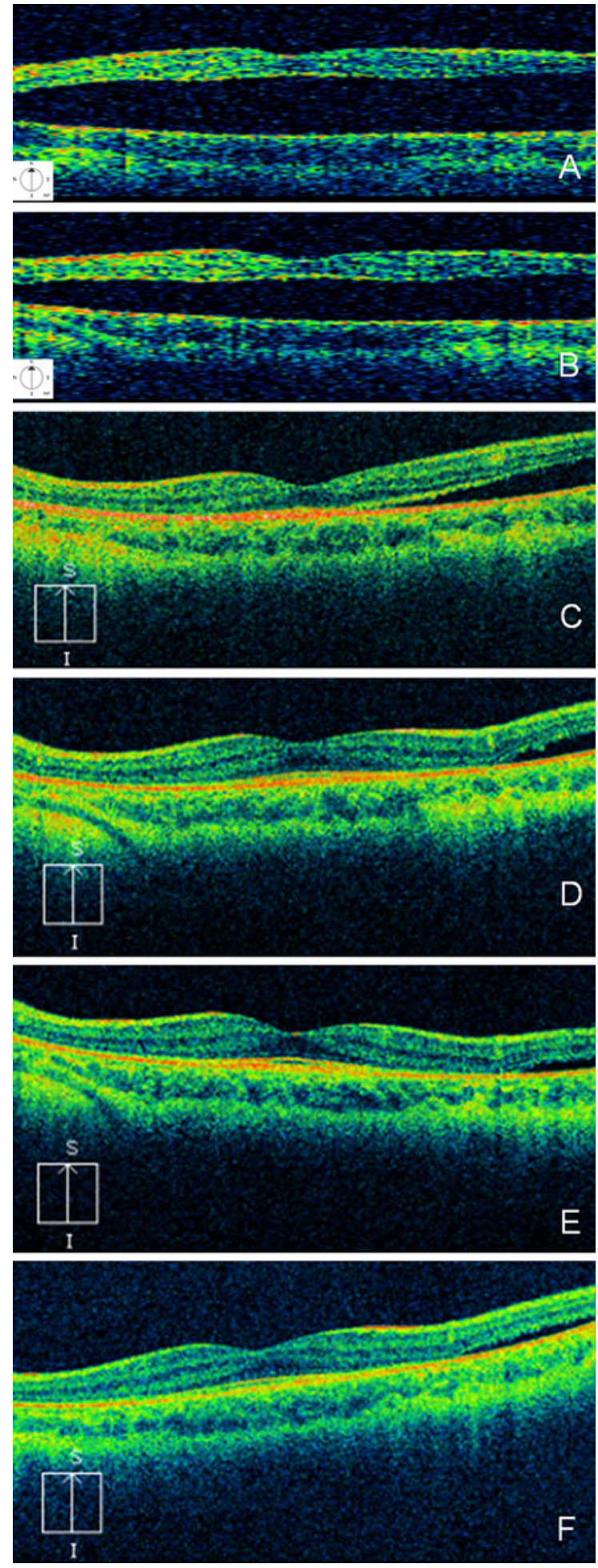

children, a progression of the macular detachment had resulted in a vitrectomy with or without peeling of the internal limiting membrane. [2, 7, 8, 16, 17].
4 Fig. 2 a-f OCT images of the left eye obtained through the fovea, vertical bottom up scan: a, Stratus OCT demonstrated the low reflective space under the neurosensory retina confirming the presence of a neurosensory retinal detachment in the macula. b, Stratus OCT scan of the neurosensory retinal detachment at 3 months showing the decrease in the neurosensory retinal detachment. c At 9 months, the OCT depicts macular reattachment with presentation of the neurosensory separation only superior to the macular in the para- and more in perifoveal area. d Cirrus OCT scan obtained one year after the first OCT depicts foveal and parafoveal retinal reattachment, but there is still a residual retinal neurosensory detachment in the superior perifoveolar region. e Cirrus OCT scan at 16 months showed further decrease in detachment. $\mathbf{f}$ The last follow-up OCT scan at 21 months revealed further shallowing of the small intraretinal schisis in the superior perifoveolar area

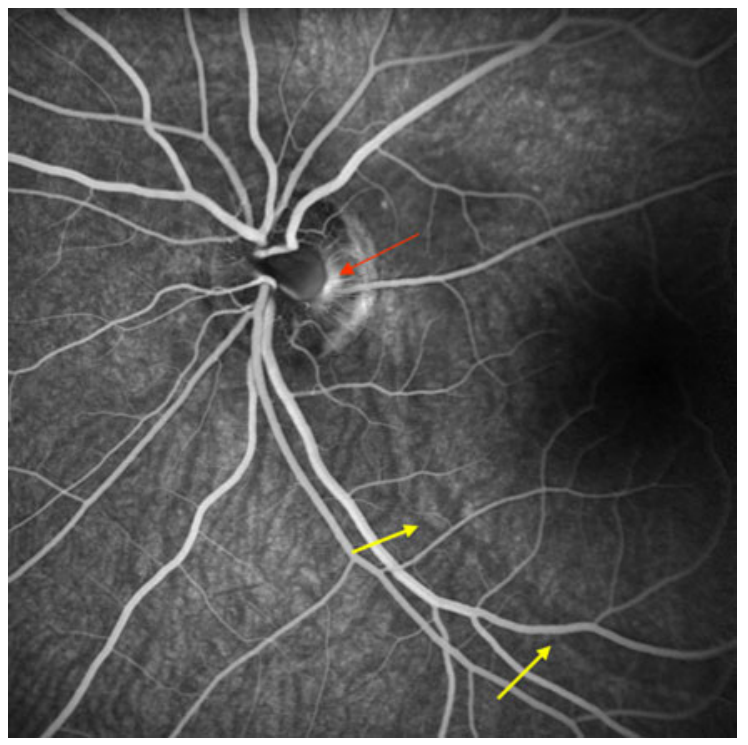

Fig. 3 Arteriovenous phase fluorescein angiogram of the left eye optic disc pit (red arrow), showing no leakage from the optic disc pit. Yellow arrows indicate the adjacent retinal elevation

Georgalas et al. [8] reported the case of a 5-year-old boy with optic disc pit maculopathy where PVD seemed intraoperatively impossible, and therefore, the posterior vitreous was peeled off from the macula area.

Despite numerous case reports with different treatment modalities offered, the best method has yet to be found, especially in children [6].

We do not have data to determine how long the condition of macular detachment had been present or whether there were previous episodes that had resolved spontaneously.

In our patient, the tendency of partial reattachment was already seen at 3 months and confirmed on OCT. 


\section{A}
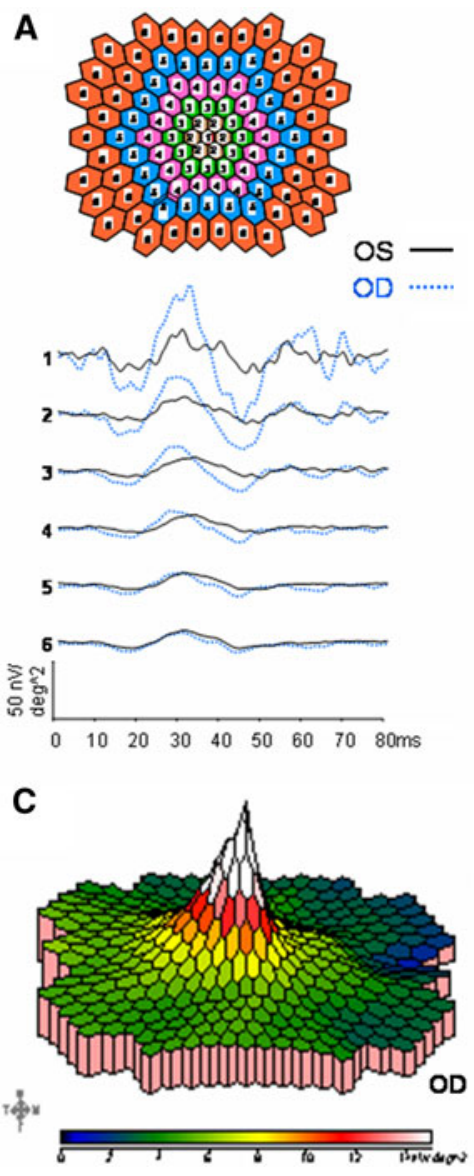

Fig. 4 a shows responses averaged in concentric rings. Responses from the right eye are shown in black and responses from the left eye in blue. Comparing the overlying the responses of the left eye to the healthy right eye, the reduction in amplitude and increase in latencies are immediately apparent. The trace

At 21 months, OCT revealed only a small residual detachment superior and nasal to the macula.

In the present case, we used the mfERG to evaluate central retinal function during the detachment and following its spontaneous reattachment. Although visual acuity had improved dramatically, there was only little improvement in the mfERG. This improvement was predominantly seen in the central $7^{\circ}$. The superior temporal area still showed most decrease in the retinal electrophysiological response (Fig. 5a, b left eye). This area corresponds to the slight visual field defect and fundus reflex reduction on ophthalmoscopy. In the OCT, the small residual serous detachment was located superior nasal.

In the temporal retina, the retinal dysfunction seen in the mfERG extends beyond the area that remained
OD

os

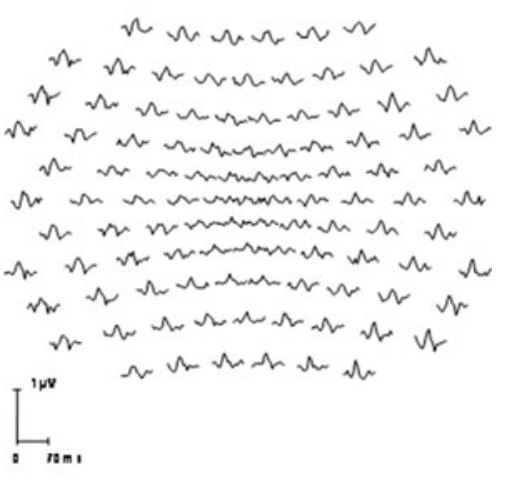

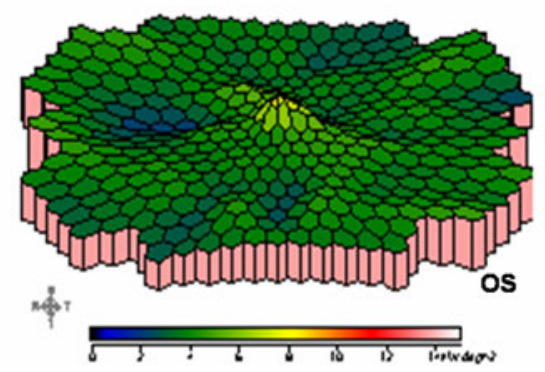

arrays (b) and topographic 3-D representation of the response density (c) of the multifocal ERG (retinal view) show a normal central peak in the right eye and a reduction in amplitude in the left eye when macula schisis associated with optic disc pit was present

affected in the OCT. This could be explained by the anatomical relation of the retinal ganglion cells (RGC) and their arcuate nerve fibre course. Retinal fluid compressing the tissue may disrupt the retrograde axonal transport and ipso facto damage the corresponding RGC bodies which are located more temporally.

There have been few reports with ODP involving children with either spontaneous resolution of the maculopathy associated with ODP [6] or those who underwent surgical treatment $[2,7]$.

The authors, who observed spontaneous resolution of serous maculopathy in a child, speculated that electrophysiological testing "would have been of value in further assessing the patient's visual function" [6]. However, the electrophysiological study of 
Fig. 5 Multifocal ERG (retinal view) after 21 months of observation. The trace arrays are shown in $\mathbf{a}$ and the topographic 3-D representation of the response density in $\mathbf{b}$ with apposition of the central retina, the central mfERG responses improved, especially in the central $7^{\circ}$
A

OD

os

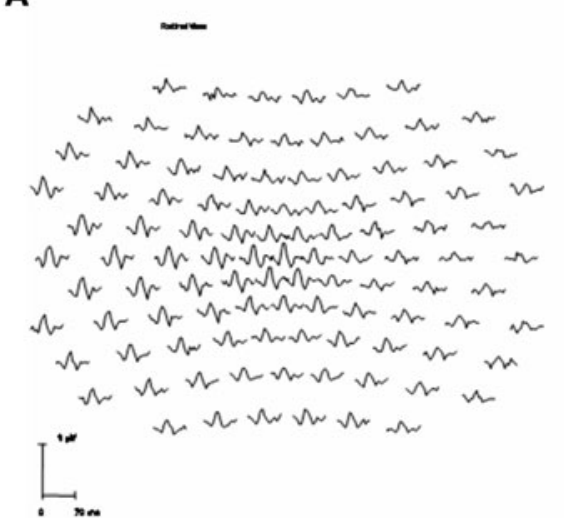

B

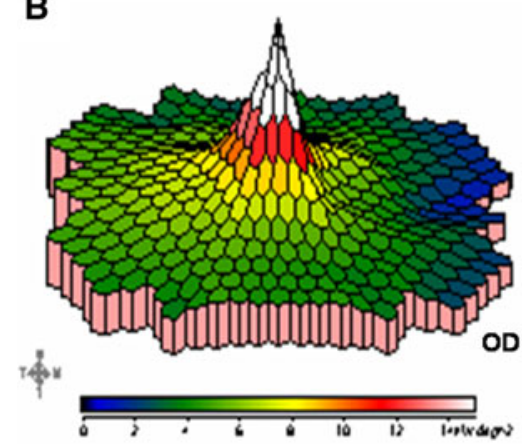

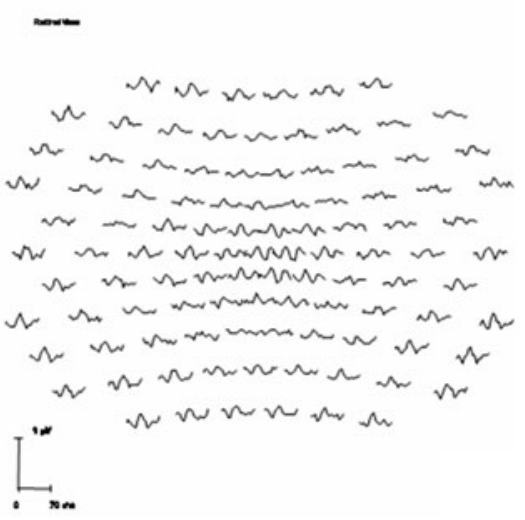

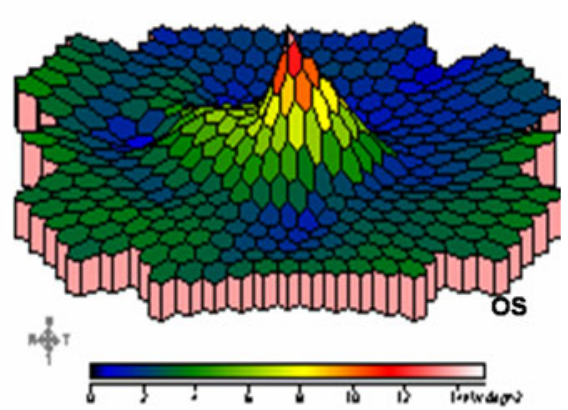

Theodossiadis et al. in 2002 showed that improvement in the electrophysiological response was not always followed by an increase in visual acuity. That is why the authors postulate that "the values of retinal response densities before treatment cannot be used alone as a prognostic factor for the postoperative functional retinal results" [1]. In contrast, in our case despite the improved visual acuity, there was still a depression in amplitude of the retinal response in the multifocal ERG of the affected eye.

Similar changes have been found in the mfERG response after recovery from central serous chorioretinopathy (CSC), where the mfERG responses improve after resolution of clinical CSC, but may remain borderline or subnormal in amplitude even with a full visual acuity [18]. These findings suggest some degree of recovery and some degree of persistent abnormality in the affected macula after the resolution of fluid.

In this case, we first described that valid mfERG responses could be elicited from a child with a central detachment of the neurosensory retina even after several months of detachment. In contrast to the OCT, which is very helpful to assess the extent of the neurosensory detachment, mfERG offers an additional tool for follow-up of retinal function in this disorder. The follow-up with the combination of morphological and functional methods helped in making the decision to avoid surgery in our patient. This may be especially important in children, where surgical intervention is rendered difficult due to the strong connection between vitreous and retina. In our case, we did not observe a macula hole, vitreous tractions or cystic lesions which are indicators of a poor visual prognosis and therefore might seem to require urgent surgical interventions.

Thus, follow-up seems a valid option in children without negative prognostic factors on OCT and with residual retinal function in the mfERG. In follow-up of children, refractive correction may need to be adapted to retinal fluid fluctuations in order to prevent a concurrent amblyopia. Better understanding of the predictive value of functional tests is needed in order not to miss the time-point where intervention becomes necessary.

Acknowledgments Swiss National Science Foundation (SNSF) 32003B-135219. 
Conflict of interest No conflicting relationship exists for any author. The author(s) have no proprietary or commercial interest in any materials discussed in the article.

\section{References}

1. Theodossiadis G, Theodossiadis P, Malias J, Moschos M, Moschos M (2002) Preoperative and postoperative assessment by multifocal electroretinography in the management of optic disc pit with serous macular detachment. Ophthalmology 109:2295-2302

2. Ghosh YK, Banerjee S, Konstantinidis A, Athanasiadis I, Kirkby GR, Tyag AK (2008) Surgical management of optic disc pit associated maculopathy. Eur J Ophthalmol 18: 142-146

3. Lincoff H, Lopez R, Kreissig I, Yannuzzi L, Cox M, Burton Th (1988) Retinoschisis associated with optic nerve pits. Arch Ophthalmol 106:61-67

4. Lincoff H, Kreissig I (1998) Optical coherence tomography in pneumatic displacement of optic disc pit maculopathy. $\mathrm{Br}$ J Ophthalmol 82:367-372

5. Brown GC, Schields JA, Goldberg RE (1980) Congenital pits of the optic nerve head. II. Clinical studies in humans. Ophthalmology 87:51-65

6. Yuen CHW, Kaye SB (2002) Spontaneous resolution of serous maculopathy associated with optic disc pit in child: a case report. J AAPOS 5:330-331

7. Ishikawa K, Terasaki H, Mori M, Sugita K, Miyake Y (2005) Optical coherence tomography before and after vitrectomy with internal limiting membrane removal in child with optic disc pit maculopathy. Jpn J Ophthalmol 49:411-413

8. Georgalas I, Kouri A, Ladas I, Gotzaridis E (2010) Optic disc pit maculopathy treated with vitrectomy, internal limiting membrane peeling, and air in a 5-year-old boy. Can J Ophthalmol 45:189-191
9. Hood DC, Bach M, Brigell M, Keating D, Kondo M, Lyons JS, Palmowski-Wolfe AM (2008) ISCEV guidelines for clinical multifocal electroretinography (2007 edition). Doc Ophthalmol 116:1-11

10. Georgalas I, Ladas I, Georgopoulos G, Petrou P (2011) Optic disc pit: a review. Graefes Arch Clin Exp Ophthalmol 249:1113-1122

11. Gass JDM (1969) Serous detachment of the macular secondary to congenital pit of the optic nerve head. Am J Ophthalmol 67:821-841

12. Irvine AR, Crawford JB, Sullivan JH (1986) The pathogenesis of retinal detachment with morning glory disk and optic pit. Retina 6:146-150

13. Ferry AP (1963) Macular detachment associated with congenital pit of the optic nerve head; pathologic findings in tow cases simulating malignant melanoma of the choroid. Arch Ophthalmol 70:346-357

14. Sugar HS (1964) An explanation for the acquired macular pathology associated with congenital pits of the optic disc. Am J Ophthalmol 57:833-835

15. Gordon R, Chatfield RK (1969) Pits in the optic disc associated with macular degeneration. Br J Ophthalmol 53:481-489

16. Hirakata A, Hida T, Wakabayashi T, Fukuda M (2005) Unusual posterior hyaloids strand in young child with optic disc pit maculopathy: intraoperative and histopathological findings. Jpn J Ophthalmol 49:264-266

17. Snead MP, James N, Jacobs PM (1991) Vitrectomy, argon laser and gas tamponade for serous retinal detachment associated with an optic disc pit: a case report. Br J Ophthalmol 75:381-382

18. Chappelow AV, Marmor M (2000) Multifocal electroretinogram abnormalities persist following resolution of central serous chorioretinopathy. Arch Ophthalmology 118:12111215 